\title{
JPE-12-4-2
}

\section{Interleaved ZVS Resonant Converter with a Parallel-Series Connection}

\author{
Bor-Ren Lin ${ }^{\dagger}$ and Sin-Jhih Shen* \\ ${ }^{\dagger *}$ Dept. of Electrical Eng., National Yunlin University of Science and Technology, Yunlin, Taiwan
}

\begin{abstract}
This paper presents an interleaved resonant converter with a parallel-series transformer connection in order to achieve ripple current reduction at the output capacitor, zero voltage turn-on for the active switches, zero current turn-off for the rectifier diodes, less voltage stress on the rectifier diodes, and less current stress on the transformer primary windings. The primary windings of the two transformers are connected in parallel in order to share the input current and to reduce the root-mean-square ( $r m s$ ) current on the primary windings. The secondary windings of the two transformers are connected in series in order to ensure that the transformer primary currents are balanced. A full-wave diode rectifier is used at the output side to clamp the voltage stress of the rectifier diode at the output voltage. Two circuit modules are operated with the interleaved PWM scheme so that the input and output ripple currents are reduced. Based on the resonant behavior, all of the active switches are turned on under zero voltage switching (ZVS), and the rectifier diodes are turned off under zero current switching (ZCS) if the operating switching frequency is less than the series resonant frequency. Finally, experiments with a $1 \mathrm{~kW}$ prototype are described to verify the effectiveness of the proposed converter.
\end{abstract}

Key words: Interleaved PWM, LLC converter, VCO, ZCS, ZVS

\section{INTRODUCTION}

To mitigate the environmental pollution and climate changes resulting from fossil fuel based power generation, soft switching power converters have been proposed recently to meet the demand for high efficiency from organizations such as the Environment Protection Agency (EPA) and the Climate Saver Computing Initiative (CSCI). There are two different kinds of control schemes related to the switching frequency in soft switching converters. One is based on a constant switching frequency with a variable duty cycle, while the other is based on a constant duty cycle and a variable switching frequency. Power converters with constant switching frequency and zero voltage switching (ZVS) techniques, such as active clamped ZVS converters [1]-[5], asymmetric half-bridge converters [6]-[7] and phase-shift pulse-width modulation (PWM) converters [8]-[9], have been proposed for many years to reduce the switching losses of MOSFETs and to increase circuit efficiency. However, the

Manuscript received Aug. 12, 2011; revised May 4, 2012

Recommended for publication by Associate Editor Kyo-Beum Lee.

${ }^{\dagger}$ Corresponding Author: linbr@yuntech.edu.tw

Tel: +886-5-5517456, Fax: +886-5-5312065, Nat'l Yunlin Univ. of Sci. and Tech.

*Dept. of Electrical Eng., National Yunlin University of Science and Technology, Taiwan
ZVS effect of these techniques is limited to specific load conditions. Thus it is difficult to design optimal converters with wide load ranges. Power converters with a variable switching frequency and the ZVS technique such as resonant converters [10]-[15] have been proposed to achieve ZVS turn-on or zero current switching (ZCS) turn-off for active switches. In a conventional series resonant converter, the output voltage cannot be properly regulated under the no-load condition due to its limited voltage gain. In order to overcome the drawbacks of conventional series resonant converters, LLC series resonant converters [16]-[19] have been presented that have the advantages of a high conversion efficiency, a high power density and a high voltage gain. If the switching frequency is less than the series resonant frequency, the rectifier diodes at the secondary side can be turned off under ZCS to overcome the reverse recovery losses.

This paper presents an interleaved series resonant converter to achieve ZVS turn-on for all of the active switches, ZCS turn-off for the rectifier diodes, ripple current reduction at the output capacitor and load current sharing. Two converter modules connected in parallel are used in the proposed circuit to share the load current and to reduce the current stress of the active switches. The interleaved PWM scheme is adopted to control the two circuit modules so that 
the ripple current at the output capacitors partially cancelled each other out. For each circuit module, a series resonant converter with two transformers and a full-wave diode rectifier is adopted to realize soft switching for all of the semiconductors. The input impedance of the resonant tank is related to the operating switching frequency. Thus the output voltage can be regulated by the variable switching frequency for different input voltages and load conditions. Since the input impedance of the resonant tank is operated at an inductive load, the active switches can be turned on under ZVS and the rectifier diodes can be turned off under ZCS if the switching frequency is less than the series resonant frequency. Thus the switching losses of the power switches and the reverse recovery losses of the rectifier diodes can be reduced. Two transformers are used in each circuit module to reduce the size of the magnetic core and to lessen the current stress on the primary windings. The secondary windings of the two transformers are connected in series in order to balance the transformer primary currents and to share the input current. For high output voltage applications, a full-wave diode rectifier is used in the secondary side to clamp the voltage stress of the rectifier diodes at the output voltage level. Compared to the conventional DC/DC converters used in low input voltage and high output voltage applications such as push-pull converters and symmetric half-bridge converters, the proposed converter has better circuit efficiency with a smaller size magnetic core and soft switching. Finally, experiments conducted on a $1 \mathrm{~kW}$ prototype to verify the effectiveness of the proposed converter are described.

\section{CIRCUIT CONFIGURATION}

The basic block of a renewable energy power conversion system is given in Fig. 1(a). The front stage is a DC/DC converter and the second stage is a DC/AC inverter. Generally the input of the front stage comes from a PV based or fuel cell based renewable energy source with a low voltage input. The output of the DC/DC converter is a high DC voltage. Then a single-phase or a three-phase inverter is used to generate AC sinusoidal voltage. The circuit configuration of the proposed DC/DC converter is given in Fig. 1(b). Since the duty cycle for each of the active switches is equal to 0.5 , the variable frequency modulation is adopted to regulate the output voltage. There are two circuit modules connected in parallel to share the input and output currents. The two circuit modules are operated with the interleaved scheme so that the input and output currents are partially cancelled. Therefore, the input and output ripple currents are reduced. Since the adopted circuit is used for high input current applications, the two transformers are connected in parallel in each of the circuit modules to share the input current. The secondary windings of the two transformers are connected in series in

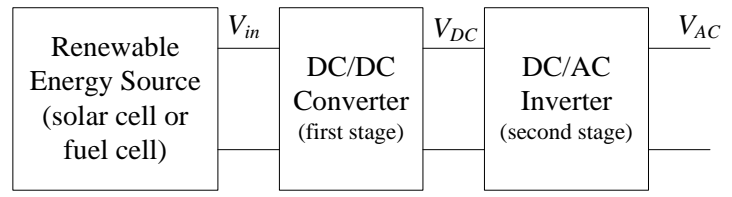

(a)

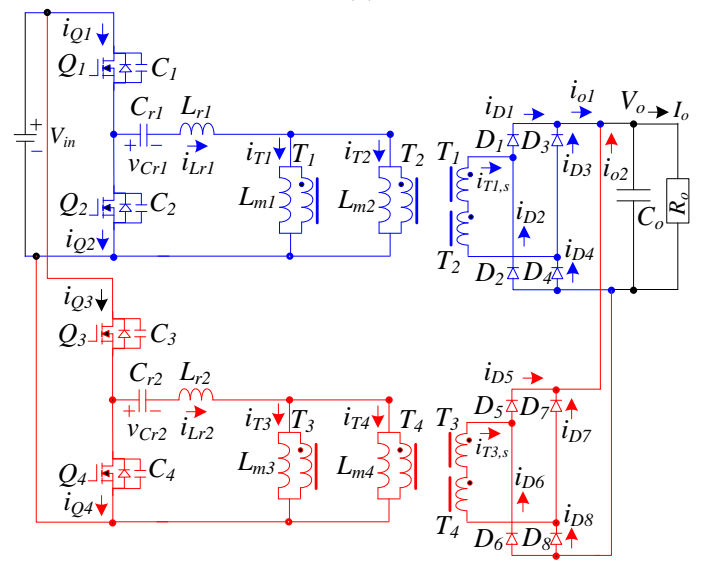

(b)

Fig. 1. Circuit configuration (a) renewable energy conversion system (b) the proposed DC/DC converter.

order to balance the two primary currents. $V_{i n}$ and $V_{o}$ are the input and output terminal voltages. The input voltage $V_{\text {in }}$ maybe a fuel cell voltage or a PV voltage from a solar panel. The first circuit module includes $Q_{1}, Q_{2}, C_{r 1}, L_{r 1}, T_{1}, T_{2}$, $D_{1} \sim D_{4}$ and $C_{0}$. The primary windings of $T_{1}$ and $T_{2}$ are connected in parallel to share the input inductor current $i_{L r 1}$. The secondary windings of $T_{1}$ and $T_{2}$ are connected in series to balance the primary currents, $i_{T 1} \approx i_{T 2} \approx i_{L r 1} / 2$. The resonant tank in the first circuit module includes $C_{r 1}, L_{r 1}, L_{m 1}$ and $L_{m 2} . D_{1} \sim D_{4}$ are the rectifier diodes. $C_{1}$ and $C_{2}$ are the output capacitances of $Q_{1}$ and $Q_{2}$, respectively. $C_{o}$ is the output capacitance. Full-wave diode rectifiers are adopted in the secondary side in order to reduce the voltage stress on the rectifier diodes for high output voltage applications. The components of the second circuit module are $Q_{3}, Q_{4}, C_{r 2}, L_{r 2}$, $T_{3}, T_{4}, D_{5} \sim D_{8}$ and $C_{0}$. The primary and secondary windings of $T_{3}$ and $T_{4}$ are connected in parallel and series respectively to share the input inductor current $i_{L r 2}$ and to balance the two primary currents $i_{T 3} \approx i_{T 4}$. The resonant tank in the second circuit module includes $C_{r 2}, L_{r 2}, L_{m 3}$ and $L_{m 4}$. Based on the series resonant phenomenon, the active switches $Q_{1} \sim Q_{4}$ are turned on under ZVS if the resonant tank is operated as an inductive load. Therefore, the switching losses on the active switches are reduced to increase the circuit efficiency. On the other hand, the rectifier diodes can be turned off under ZCS if the switching frequency of the proposed converter is less than the series resonant frequency under a full load and a maximum input voltage. Thus the reverse recovery losses on fast recovery diodes are reduced. The proposed converter can be used for low input voltage and high output voltage 


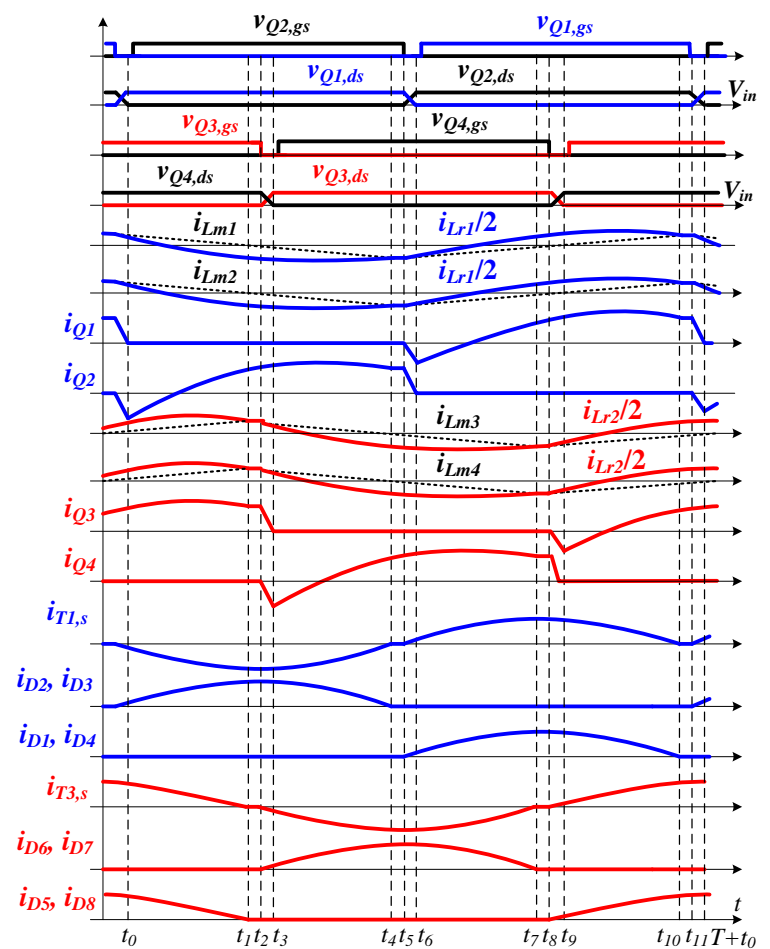

Fig. 2. Key waveforms of the proposed converter.

applications such as the battery-based DC/DC converters, the high power discharger systems and the DC/DC converters used in renewable energy systems.

\section{OPERATION PRINCIPLE AND SYSTEM ANALYSIS}

Each switch in the proposed converter is turned on with a $50 \%$ duty ratio. Therefore the output voltage regulation is controlled by a frequency modulation technique. The components of the two circuit modules are identical. The four transformers have the same turns ratio $\left(n=n_{p} / n_{s}\right)$ and the same magnetizing inductance $L_{m}$. All of the active switches are ideal and have the same output capacitance $\left(C_{1}=C_{2}=C_{3}=C_{4}=C_{e q}\right)$. Fig. 2 shows the key waveforms during a switching cycle. There are twelve operating modes in the proposed converter for each switching period. The equivalent circuits for each of the operating modes are shown in Fig. 3. Before time $t_{0}, Q_{3}, D_{2}, D_{3}, D_{5}$ and $D_{8}$ conduct. $C_{1}$ is charged and $C_{2}$ is discharged.

Mode 1 [ $t_{0} \leq t<t_{1}$ ]: At time $t_{0}, C_{2}$ is discharged to zero voltage. Since $i_{Q 2}<0$, the anti-parallel diode of $Q_{2}$ conducts. Switch $Q_{2}$ can be turned on at this moment to achieve ZVS. Since $i_{T 1}<i_{L m 1}, i_{T 2}<i_{L m 2}, i_{T 3}>i_{L m 3}$ and $i_{T 4}>i_{L m 4}$, diodes $D_{2}, D_{3}, D_{5}$ and $D_{8}$ conduct in this mode so that $v_{L m 1}=v_{L m 2}=-n V_{o} / 2$ and $v_{L m 3}=v_{L m 4}=n V_{o} / 2$. The magnetizing currents $i_{L m 1}$ and $i_{L m 2}$ decrease linearly with a slope of $-n V_{o} /\left(2 L_{m}\right)$ and the magnetizing currents $i_{L m 3}$ and $i_{L m 4}$ increase linearly with a slope of $n V_{o} /\left(2 L_{m}\right) . C_{r 1}$ and $L_{r 1}$ are resonant with the applied voltage $n V_{o} / 2$ in circuit module 1 , while $C_{r 2}$ and $L_{r 2}$ are resonant with the applied voltage $V_{i n}-n V_{o} / 2$ in circuit module 2. $i_{L r 1}$ and $v_{C r 1}$ decrease, and $i_{L r 2}$ and $v_{C r 2}$ increase in this mode. The resonant inductor currents $i_{L r 1}$ and $i_{L r 2}$ and the capacitor voltages $v_{C r 1}$ and $v_{C r 2}$ in this mode are expressed as:

$$
\begin{aligned}
i_{L r 1}(t)= & \frac{n V_{o} / 2-v_{C r 1}\left(t_{0}\right)}{Z_{r}} \sin \omega_{r}\left(t-t_{0}\right)+i_{L r 1}\left(t_{0}\right) \cos \omega_{r}\left(t-t_{0}\right) \\
i_{L r 2}(t)= & \frac{V_{i n}-n V_{o} / 2-v_{C r 2}\left(t_{0}\right)}{Z_{r}} \sin \omega_{r}\left(t-t_{0}\right) \\
& +i_{L r 2}\left(t_{0}\right) \cos \omega_{r}\left(t-t_{0}\right) \\
v_{C r 1}(t)= & n V_{o} / 2-\left[n V_{o} / 2-v_{C r 1}\left(t_{0}\right)\right] \cos \omega_{r}\left(t-t_{0}\right) \\
& +i_{L r 1}\left(t_{0}\right) Z_{r} \sin \omega_{r}\left(t-t_{0}\right) \\
v_{C r 2}(t)= & V_{i n}-n V_{o} / 2-\left[V_{i n}-n V_{o} / 2-v_{C r 2}\left(t_{0}\right)\right] \times \\
& \cos \omega_{r}\left(t-t_{0}\right)+i_{L r 2}\left(t_{0}\right) Z_{r} \sin \omega_{r}\left(t-t_{0}\right)
\end{aligned}
$$

where $Z_{r}=\sqrt{L_{r} / C_{r}}$ and $\omega_{r}=1 / \sqrt{L_{r} C_{r}}$. Power is transferred from the input terminal voltage $V_{\text {in }}$ to the output load through $Q_{3}, C_{r 2}, L_{r 2}, T_{3}, T_{4}, D_{5}$ and $D_{8}$. This mode ends at time $t_{1}$ when $i_{L m 3}=i_{T 3}$ and $i_{L m 4}=i_{T 4}$. Then diodes $D_{5}$ and $D_{8}$ are off.

Mode $2\left[t_{1} \leq t<t_{2}\right]$ : This mode starts at $t_{1}$ when $i_{L m 3}=i_{T 3}$ and $i_{L m 4}=i_{T 4}$. Then diodes $D_{5}$ and $D_{8}$ are off in circuit module 2 . Since $Q_{3}$ still conducts in this mode, $C_{r 2}, L_{r 2}, L_{m 3}$ and $L_{m 4}$ are resonant in circuit module 2 . On the other hand, $C_{r 4}, L_{r 4}$ and $L_{m 4}$ are also resonant with the input voltage $V_{i n} / 2 . i_{L r 2}$ and $v_{C r 2}$ are obtained as:

$$
\begin{gathered}
i_{L r 2}(t)=\frac{V_{i n}-v_{C r 2}\left(t_{1}\right)}{Z_{p}} \sin \omega_{p}\left(t-t_{1}\right)+i_{L r 2}\left(t_{1}\right) \cos \omega_{p}\left(t-t_{1}\right) \\
v_{C r 2}(t)=V_{i n}-\left[V_{i n}-v_{C r 2}\left(t_{1}\right)\right] \cos \omega_{p}\left(t-t_{1}\right) \\
+i_{L r 2}\left(t_{1}\right) Z_{p} \sin \omega_{p}\left(t-t_{1}\right)
\end{gathered}
$$

where $Z_{p}=\sqrt{\frac{L_{r}+L_{m} / 2}{C_{r}}}$ and $\omega_{r}=1 / \sqrt{\left(L_{r}+L_{m} / 2\right) C_{r}}$.

The operation of circuit module 1 in this mode is the same as the operation in mode 1.

Mode 3 [ $\left.t_{2} \leq t<t_{3}\right]$ : At time $t_{2}, Q_{3}$ is turned off and diodes $D_{6}$ and $D_{7}$ conduct. Thus $v_{L m 3}=v_{L m 4}=-n V_{o} / 2$ and $i_{L m 3}$ and $i_{L m 4}$ decrease in this mode. Since $i_{L r 2}\left(t_{2}\right)>0, C_{3}$ is charged and $C_{4}$ is discharged. If the energy stored in $L_{r 2}$ at time $t_{2}$ is greater than the energy stored in $C_{3}$ and $C_{4}$, then $C_{4}$ can be discharged to zero voltage at time $t_{3}$. The capacitor voltages $v_{C 3}$ and $v_{C 4}$ are expressed as:

$$
\begin{aligned}
& v_{C 3}(t) \approx i_{L r 2}\left(t_{2}\right)\left(t-t_{2}\right) /\left(2 C_{e q}\right) \\
& v_{C 4}(t) \approx V_{i n}-i_{L r 2}\left(t_{2}\right)\left(t-t_{2}\right) /\left(2 C_{e q}\right)
\end{aligned}
$$

The operation behavior of circuit module 1 is the same as the circuit operation in mode 2.

Mode $4\left[t_{3} \leq t<t_{4}\right]$ : At time $t_{3}, C_{4}$ is discharged to zero voltage so that the anti-parallel diode of $Q_{4}$ conducts. Before $i_{Q 4}$ becomes positive, $Q_{4}$ is turned on under ZVS. Since $D_{2}, D_{3}$, 
$D_{6}$ and $D_{7}$ conduct in this mode, the magnetizing voltages $v_{L m 1}=v_{L m 2}=v_{L m 3}=v_{L m 4}=-n V_{o} / 2$. Thus $i_{L m 1} \sim i_{L m 4}$ decrease in this mode. $C_{r 1}$ and $L_{r 1}$ are resonant in circuit module 1 , while $C_{r 2}$ and $L_{r 2}$ are resonant in circuit module 2 .

Mode $5\left[t_{4} \leq t<t_{5}\right]$ : At time $t_{4}, i_{L m 1}=i_{T 1}$ and $i_{L m 2}=i_{T 2}$ in circuit module 1 such that diodes $D_{1} \sim D_{4}$ are reverse biased. Since $Q_{2}$ still conducts, $C_{r 1}, L_{r 1}, L_{m 1}$ and $L_{m 2}$ are resonant in this mode. The operation behavior of circuit module 2 is the same as the circuit operation in mode 4.

Mode $6\left[t_{5} \leq t<t_{6}\right]$ : At time $t_{5}, Q_{2}$ turns off and diodes $D_{1}$ and $D_{4}$ conduct so that $v_{L m 1}=v_{L m 2}=n V_{o} / 2$. Therefore, $i_{L m 1}$ and $i_{L m 2}$ increase in this mode. Since $i_{L r 1}\left(t_{5}\right)<0, C_{1}$ is discharged and $C_{2}$ is charged. If the energy stored in $L_{r 1}$ at time $t_{5}$ is greater than the energy stored in $C_{1}$ and $C_{2}$, then $C_{1}$ can be discharged to zero voltage at time $t_{6}$. The operation behavior of circuit module 2 in this mode is the same as the operation in mode 5.

Mode 7 [ $\left.t_{6} \leq t<t_{7}\right]$ : At time $t_{6}, C_{1}$ is discharged to zero voltage. Since $i_{Q 1}<0$, the anti-parallel diode of $Q_{1}$ conducts. Therefore, $Q_{1}$ can be turned on at this moment to achieve ZVS. Since $D_{1}$, $D_{4}, D_{6}$ and $D_{7}$ conduct in this mode, $v_{L m 1}=v_{L m 2}=n V_{0} / 2$ and $v_{L m 3}=v_{L m 4}=-n V_{o} / 2$ so that $i_{L m 1}$ and $i_{L m 2}$ increase, while $i_{L m 3}$ and $i_{L m 4}$ decrease. $L_{r 1}$ and $C_{r 1}$ are resonant with the applied voltage $V_{i n}-n V_{o} / 2$ in circuit module 1 , while $L_{r 2}$ and $C_{r 2}$ are resonant with the applied voltage $n V_{o} / 2$ in circuit module 2 .

Mode $8\left[t_{7} \leq t<t_{8}\right]$ : At time $t_{7}, i_{L m 3}=i_{T 3}$ and $i_{L m 4}=i_{T 4}$ so that diodes $D_{5} \sim D_{8}$ are off. Since $Q_{4}$ still conducts, $C_{r 2}, L_{r 2}, L_{m 3}$ and $L_{m 4}$ are resonant in module 2 . The circuit operation of circuit module 1 is the same as the circuit operation in mode 7.

Mode $9\left[t_{8} \leq t<t_{9}\right]$ : At time $t_{8}, Q_{4}$ is turned off and diodes $D_{5}$ and $D_{8}$ conduct. Therefore, $v_{L m 3}=v_{L m 4}=n V_{o} / 2$ and the magnetizing currents $i_{L m 3}$ and $i_{L m 4}$ increase in this mode. Since $i_{L r 2}\left(t_{8}\right)<0, C_{4}$ is charged and $C_{3}$ is discharged. If the energy stored in $L_{r 2}$ at time $t_{8}$ is greater than the energy stored in $C_{3}$ and $C_{4}$, then $C_{3}$ can be discharged to zero voltage at time $t_{9}$. The operation behavior of circuit module 1 in this mode is the same as the circuit operation in mode 8 .

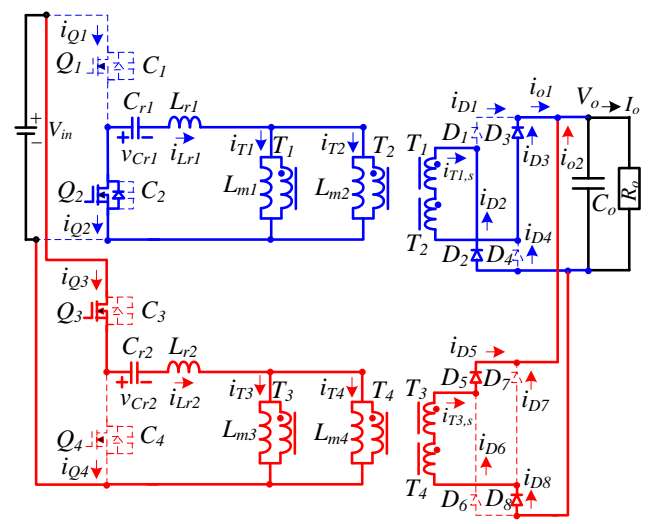

(a)

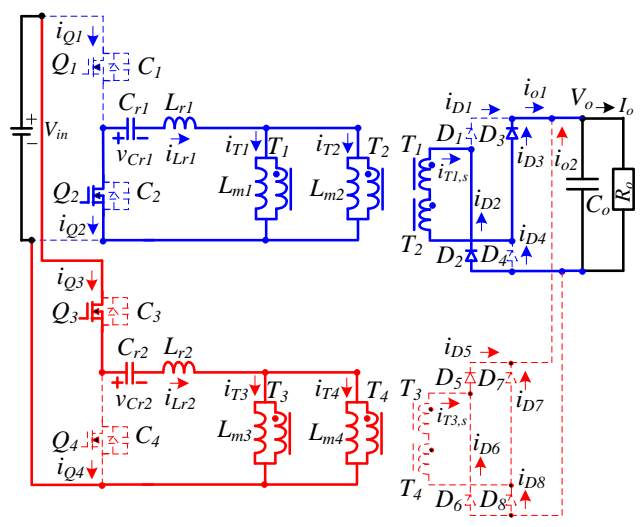

(b)

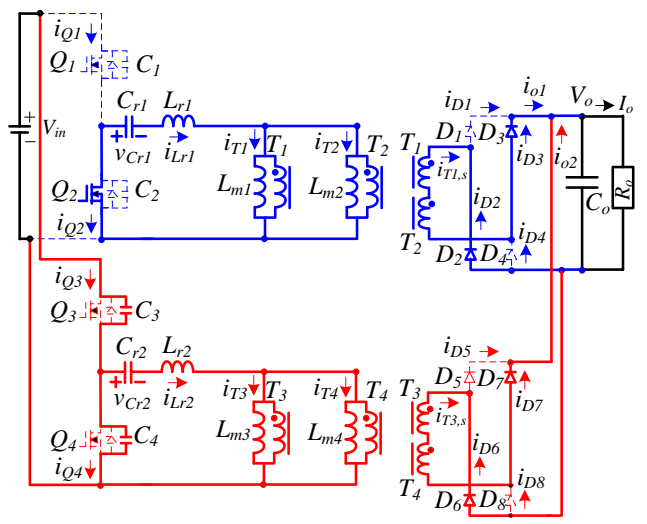

(c)

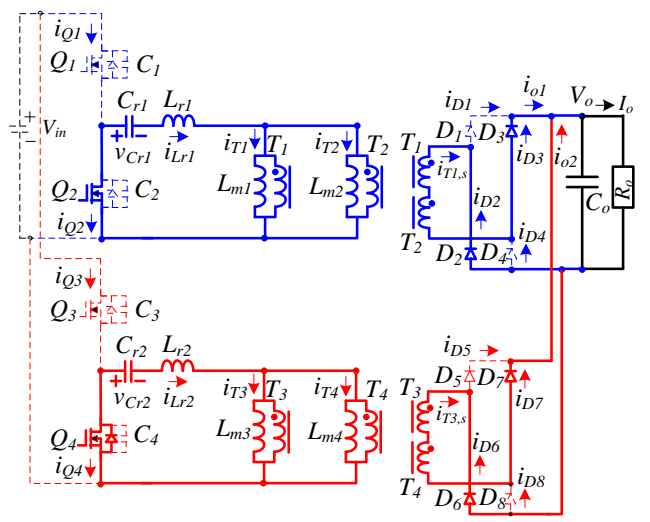

(d)

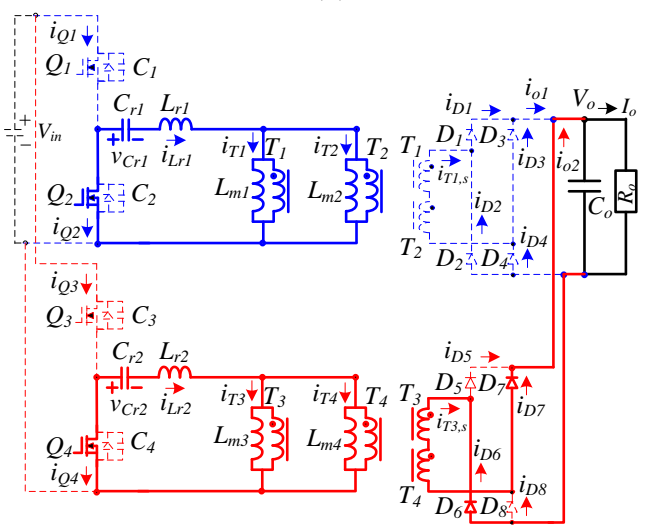

(e) 
532

Journal of Power Electronics, Vol. 12, No. 4, July 2012

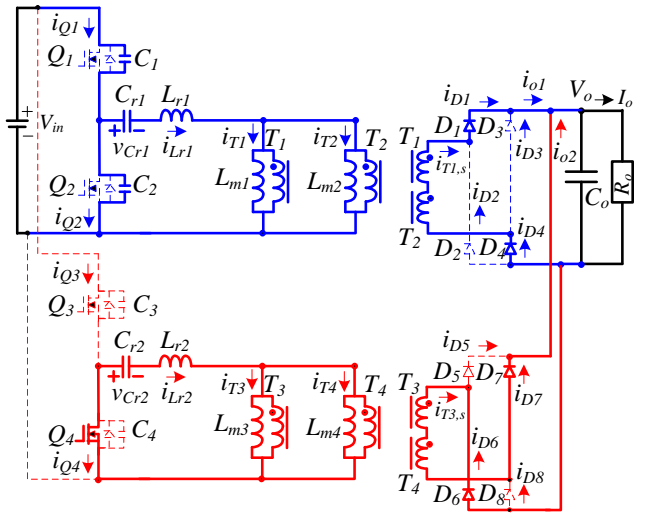

(f)

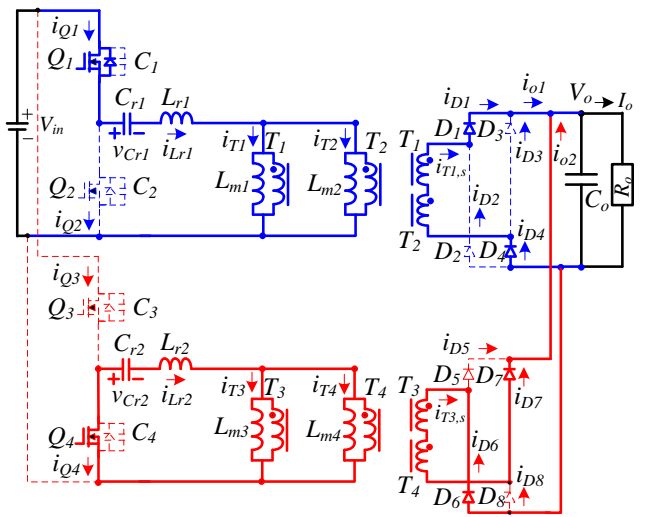

(g)

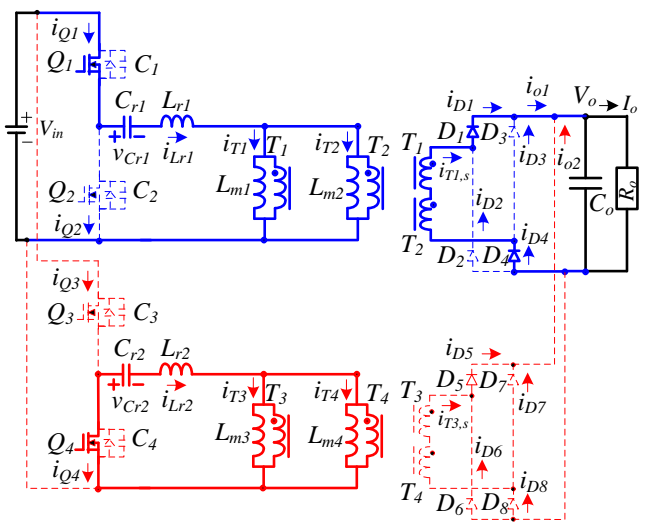

(h)

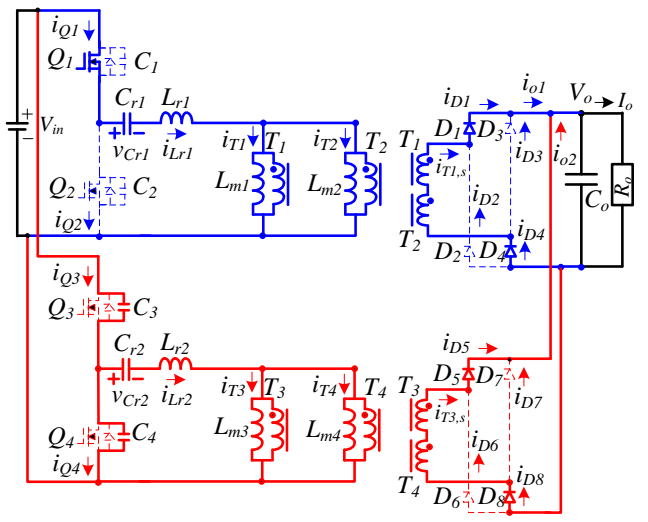

(i)

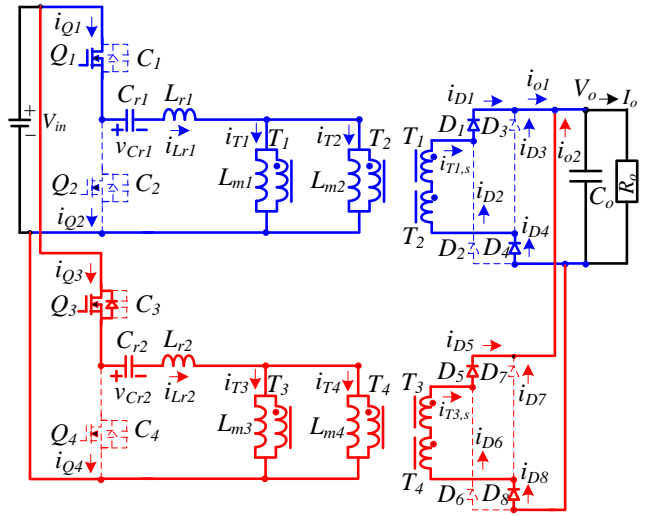

(j)

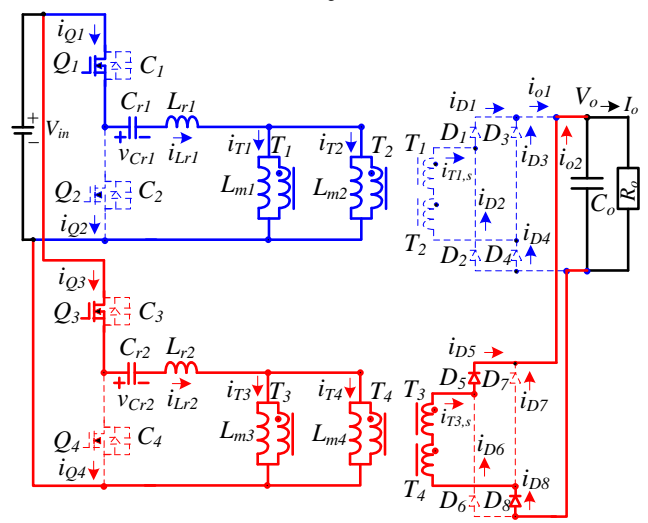

(k)

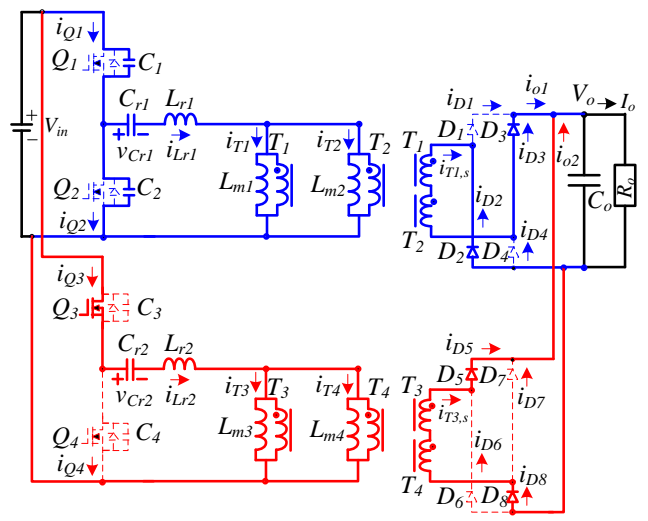

(l)

Fig. 3 Operation modes of the proposed converter during one switching cycle (a) mode 1 (b) mode 2 (c) mode 3 (d) mode 4 (e) mode 5 (f) mode 6 (g) mode 7 (h) mode 8 (i) mode 9 (j) mode 10 (k) mode 11 (l) mode 12.

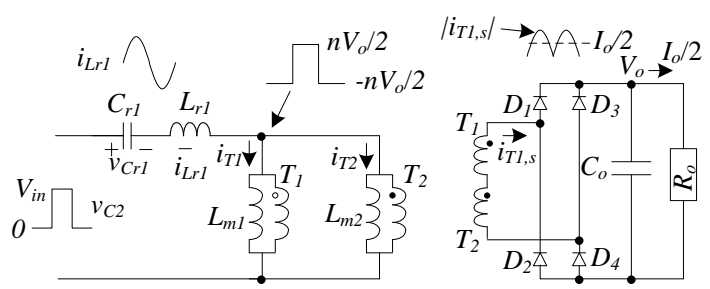

Fig. 4. Equivalent circuit of circuit module 1. 
Mode $10\left[t_{9} \leq t<t_{10}\right]$ : At time $t_{9}$, capacitor $C_{3}$ is discharged to zero voltage. Since $i_{L r 2}\left(t_{9}\right)<0$, the anti-parallel diode of $Q_{3}$ conducts. Thus $Q_{3}$ can be turned on at this moment to achieve ZVS. Diodes $D_{1}, D_{4}, D_{5}$ and $D_{8}$ conduct in this mode so that $v_{L m 1}=v_{L m 2}=v_{L m 3}=v_{L m 4}=n V_{o} / 2$. The magnetizing currents $i_{L m 1} \sim i_{L m 4}$ increase in this mode. Input power is delivered to the output load through $Q_{1}, C_{r 1}, L_{r 1}, T_{1}, T_{2}, D_{1}$ and $D_{4}$ in circuit module 1 and through $Q_{3}, C_{r 2}, L_{r 2}, T_{3}, T_{4}, D_{5}$ and $D_{8}$ in circuit module 2. Therefore, $i_{L r 1}, i_{L r 2}, v_{C r 1}$ and $v_{C r 2}$ increase.

Mode $11\left[t_{10} \leq t<t_{11}\right]$ : At time $t_{10}, i_{L m 1}=i_{T 1}$ and $i_{L m 2}=i_{T 2}$ so that diodes $D_{1} \sim D_{4}$ are all off in circuit module 1 . Since $Q_{1}$ still conducts, $C_{r 1}, L_{r 1}, L_{m 1}$ and $L_{m 2}$ are resonant with the applied voltage $V_{\text {in }}$. The operation behavior of circuit module 2 in this mode is the same as the circuit operation in mode 10.

Mode $12\left[t_{11} \leq t<T+t_{0}\right]$ : At time $t_{11}, Q_{1}$ is turned off and diodes $D_{2}$ and $D_{3}$ conduct. Thus $v_{L m 1}=v_{L m 2}=-n V_{o} / 2$. The magnetizing currents $i_{L m 1}$ and $i_{L m 2}$ decrease in this mode. Since $i_{L r 1}\left(t_{11}\right)>0$, $\mathrm{C}_{1}$ is charged and $C_{2}$ is discharged. If the energy stored in $L_{r 1}$ at time $t_{11}$ is greater than the energy stored in $C_{1}$ and $C_{2}$, then $C_{2}$ can be discharged to zero voltage at time $T+t_{0}$. Then the anti-parallel diode of $Q_{2}$ conducts. This is the last operating mode of the proposed converter during one switching period.

The power flow through the resonant tank is related to the switching frequency. All of the harmonics of the switching frequency are neglected in the following analysis. Fig. 4 shows an equivalent circuit of circuit module 1 . The duty ratios of switches $Q_{1}$ and $Q_{2}$ are equal to 0.5 . The input voltage of the resonant tank is a square waveform between 0 and $V_{i n}$. The DC value and the fundamental root-mean-square (rms) value of the input voltage $v_{C 2}$ are $V_{\text {in }} / 2$ and $\sqrt{2} V_{\text {in }} / \pi$, respectively. The output side of circuit module 1 is driven by a quasi-sinusoidal current. When inductor current $i_{T 1}>i_{L m 1}, D_{1}$ and $D_{4}$ conduct and $v_{L m 1}=v_{L m 2}=n V_{o} / 2$. If $i_{T 1}<i_{L m 1}$, then $D_{2}$ and $D_{3}$ conduct and $v_{L m 1}=v_{L m 2}=-n V_{o} / 2$. Thus the fundamental rms value of the magnetizing voltage is expressed as $v_{L m 1, r m s}=\sqrt{2} n V_{o} / \pi$. Since the output current of circuit module 1 is equal to $I_{o} / 2$, the rms value of the secondary winding current is equal to $i_{T 1, s, r m s}=\pi I_{o} /(4 \sqrt{2})$. Thus the load resistance $R_{o}$ reflected to the transformer primary side is given as:

$$
R_{a c 1}=R_{a c 2}=\frac{v_{L m 1, r m s}}{i_{T 1, s, r m s} / n}=\frac{8 n^{2}}{\pi^{2}} R_{o}
$$

The AC resonant tank ( $L_{r 1}$ and $\left.C_{r 1}\right)$, shown in Fig. 5, is excited by an effectively sinusoidal input voltage $v_{C 2, f}$ and it drives the effective resistive loads $R_{a c 1}$ and $R_{a c 2}$ in circuit module 1 . The AC voltage gain of circuit module 1 , based on Fig. 5, is expressed in (10).

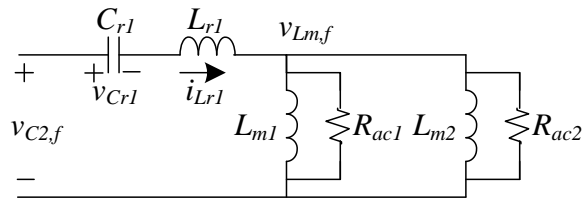

Fig. 5. AC resonant tank of circuit module 1 with fundamental switching frequency.

\section{TABLE I}

Key Circuit Parameters Of The Prototype Circuit

\begin{tabular}{|c|c|}
\hline Input voltage $V_{\text {in }}$ & $42 \mathrm{~V} \sim 52 \mathrm{~V}$ \\
\hline Output voltage $V_{o} /$ Current $I_{o}$ & $400 \mathrm{~V} / 2.5 \mathrm{~A}$ \\
\hline Series resonant frequency $f_{r}$ & $100 \mathrm{kHz}$ \\
\hline Switches $Q_{1} \sim Q_{4}$ & IRFP064 \\
\hline Diodes $D_{1} \sim D_{8}$ & $30 \mathrm{ETH} 06$ \\
\hline Turns ratio of $T_{1} \sim T_{4}$ & $n_{p}: n_{s}=6: 42$ \\
\hline Resonant inductances $L_{r 1}, L_{r 2}$ & $0.633 \mu \mathrm{H}$ \\
\hline Magnetizing inductances $L_{m 1} \sim L_{m 4}$ & $7.6 \mu \mathrm{H}$ \\
\hline Resonant capacitances $C_{r 1}, C_{r 2}$ & $4 \mu \mathrm{F}$ \\
\hline Output capacitor $C_{o}$ & $1320 \mu \mathrm{F} / 450 \mathrm{~V}$ \\
\hline
\end{tabular}
$\left|G_{a c}(f)\right|=1 / \sqrt{\left[1+k\left(1-\frac{f_{r}{ }^{2}}{f_{s}{ }^{2}}\right)\right]^{2}+Q^{2}\left(\frac{f_{s}}{f_{r}}-\frac{f_{r}}{f_{s}}\right)^{2}}$

where $f_{r}=1 /\left(2 \pi \sqrt{L_{r 1} C_{r 1}}\right), Q=\sqrt{L_{r 1} / C_{r 1}} /\left(R_{a c 1} / 2\right)$, $k=2 L_{r 1} / L_{m 1}$ and $f_{s}$ is the switching frequency. The AC voltage gain is related to the switching frequency in (10). Therefore, the output voltage $V_{o}$ can be regulated by the variation of the switching frequency $f_{s}$. Under the no load condition, $R_{a c 1}$ is infinite and the quality factor $Q$ equals zero. Then the AC voltage gain under the no load condition is expressed as: $\left|G_{a c}(f)\right|_{Q=0}=1 /\left[1+k\left(1-\frac{f_{r}^{2}}{f_{s}^{2}}\right)\right]$.

If the design minimum DC voltage gain at the maximum input voltage and the maximum switching frequency is greater than the AC voltage gain under the no load condition, then the output voltage $V_{o}$ can be controlled.

$$
\begin{aligned}
G_{d c, \text { min }} & =\frac{n\left(V_{o}+2 V_{f}\right)}{V_{i n, \max }}>1 /\left[1+k\left(1-\frac{f_{r}^{2}}{f_{s, \text { max }}^{2}}\right)\right] \\
& =\left|G_{a c}(f)\right|_{Q=0, f_{s, \max }}
\end{aligned}
$$

where $V_{f}$ is the voltage drop across diodes $D_{1} \sim D_{4}$ and $f_{s, \max }$ is the maximum switching frequency generated by the voltage controlled oscillator.

\section{EXPERIMENTAL RESULTS}




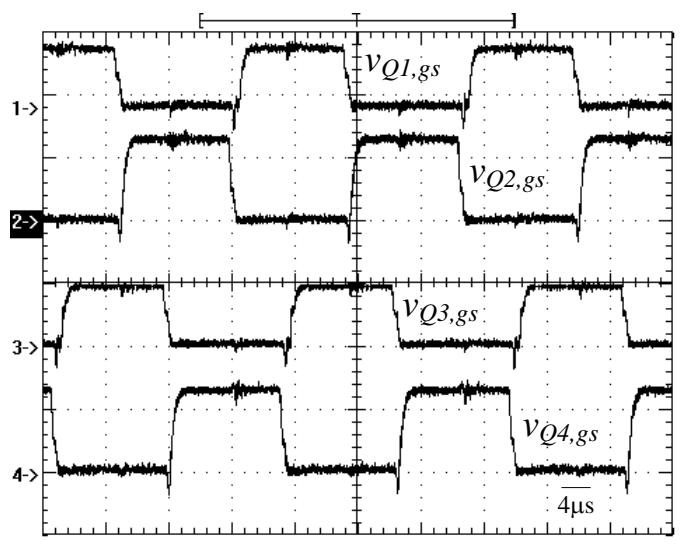

(a)

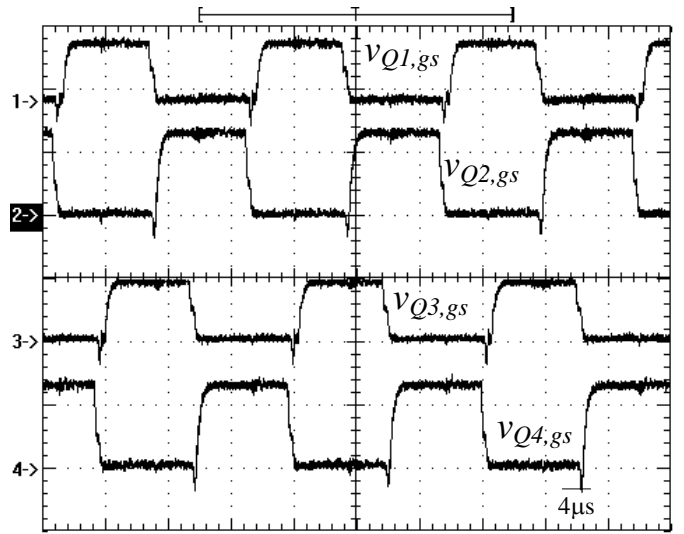

(b)

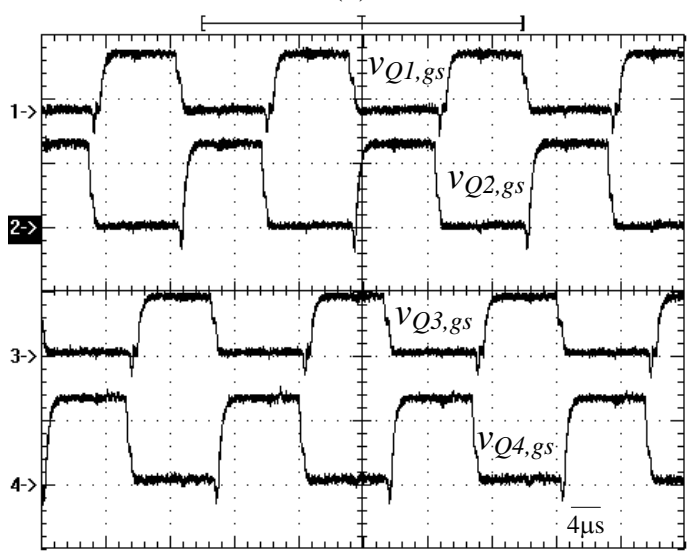

(c)

Fig. 6. results of gate voltages of $Q_{1} \sim Q_{4}$ at full load and input $\begin{array}{llll}\text { voltage (a) } V_{\text {in }}=42 \mathrm{~V} & \text { (b) } V_{\text {in }}=48 \mathrm{~V} & \text { (c) } V_{\text {in }}=52 \mathrm{~V}\end{array}$ $\left[v_{Q 1, g s} \sim v_{Q 4, g s}: 10 \mathrm{~V} / \mathrm{div}\right]$.

Experimental results based on a laboratory prototype have been provided to demonstrate the performance of the proposed converter. The circuit parameters of the prototype circuit are shown in Table I. The variable switching frequency is adopted to generate the gate signals. The flip-flop circuits are used to generate the interleaved PWM signals. First, an LLC control IC based on a L6599 is used to generate two PWM signals. Then, two D-type flip-flop ICs

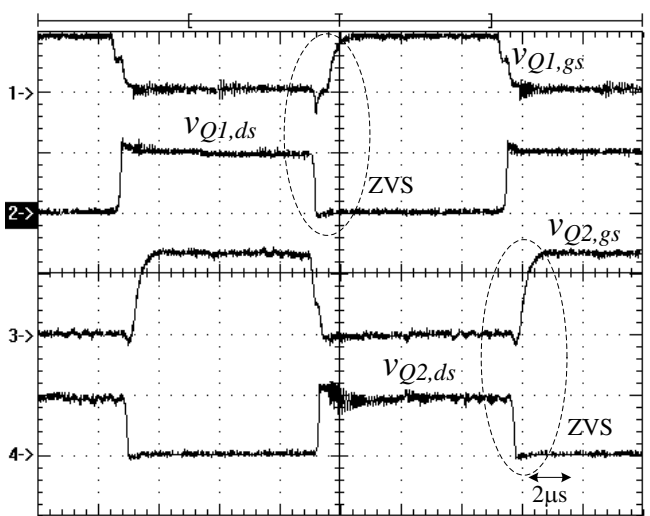

(a)

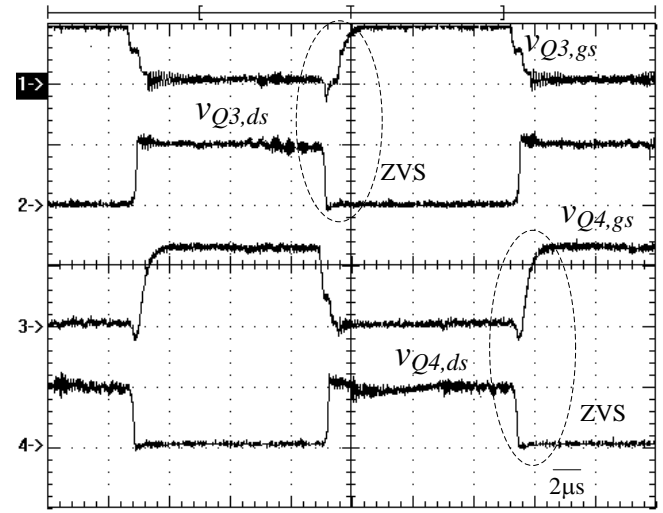

(b)

Fig. 7. Measured gate voltage and drain voltage at $V_{i n}=48 \mathrm{~V}$ and full load condition (a) $Q_{1}$ and $Q_{2}$ (b) $Q_{3}$ and $Q_{4}$ $\left[v_{Q 1, g s} \sim v_{Q 4, g s}: 10 \mathrm{~V} / \mathrm{div} ; v_{Q 1, d s} \sim v_{Q 4, d s}: 50 \mathrm{~V} / \mathrm{div}\right]$.

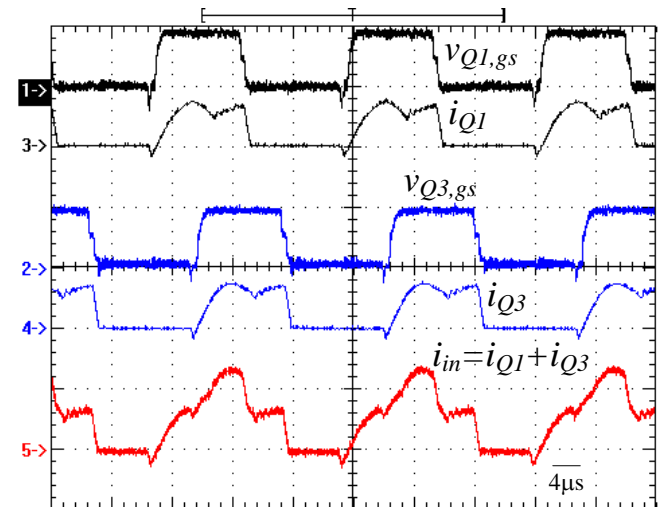

Fig. 8. Measured results of gate voltages, $v_{Q 1, g s}$ and $v_{Q 3, g s}$, switch currents, $i_{Q 1}$ and $i_{Q 3}$, and the DC input current $i_{\text {in }}$ at $V_{\text {in }}=48 \mathrm{~V}$ and full load condition $\left[v_{Q 1, g s}, v_{Q 3, g s}: 10 \mathrm{~V} / \mathrm{div} ; i_{Q 1}, i_{Q 3}\right.$, $\left.i_{i n}: 50 \mathrm{~A} / \mathrm{div}\right]$.

are used to generate four PWM signals. Fig. 6 gives the experimental results for the gate voltages of $Q_{1} \sim Q_{4}$ at different input voltages and the full load condition. It can be observed that the gate voltage $v_{Q 3, g s}$ lags the gate voltage $v_{Q 1, g s}$ by one fourth of a switching period, and that the low input 
voltage has less switching frequency. The measured gate voltage and the drain voltage of $Q_{1} \sim Q_{4}$ at an input voltage of $V_{\text {in }}=48 \mathrm{~V}$ and the full load condition are given in Fig. 7 . Before $Q_{1} \sim Q_{4}$ are turned on, the drain voltages have been decreased to zero voltage. Therefore switches $Q_{1} \sim Q_{4}$ are turned on under ZVS from $20 \%$ to $100 \%$ load conditions. Fig. 8 gives the measured waveforms of the gate voltages, $v_{Q 1, g s}$ and $v_{Q 3, g s}$, the switch currents, $i_{Q 1}$ and $i_{Q 3}$, and the DC input current $i_{\text {in }}$ at $V_{\text {in }}=48 \mathrm{~V}$ and the full load condition. Since the currents $i_{Q 1}$ and $i_{Q 3}$ are phase-shifted by one-fourth of a switching period, the input current $i_{i n}$ has less current ripple. Fig. 9 shows the measured resonant inductor currents, $i_{L r 1}$ and $i_{L r 2}$, and the resonant capacitor voltages, $v_{C r 1}$ and $v_{C r 2}$ at the full load condition. Fig. 10 gives the measured waveforms of $i_{L r 1}, i_{T 1}$ and $i_{T 2}$ for converter module 1 and $i_{L r 2}, i_{T 3}$ and $i_{T 4}$ for converter module 2 at the full load condition. The measured transformer primary currents are balanced so that $i_{T 1}=i_{T 2} \approx i_{L r 1} / 2$ and $i_{T 3}=i_{T 4} \approx i_{L r 2} / 2$. Fig. 11 shows the measured waveforms of the gate voltages, $v_{Q 1, g s}$ and $v_{Q 3, g s}$, and the diode currents $i_{D 1}, i_{D 3}, i_{D 5}$ and $i_{D 7}$ at $V_{i n}=48 V$ and the full load condition. Before switches $Q_{1} \sim Q_{4}$ are turned off, the rectifier diode currents are decreased to zero. Thus diodes $D_{1} \sim D_{8}$ are all turned off under ZCS. Therefore, the reverse recovery losses of the rectifier diodes are reduced. Fig. 12 gives the measured results of the output currents $i_{D 1}+i_{D 3}$ and $i_{D 5}+i_{D 7}$ of the two circuit modules, the resultant output current $i_{D 1}+i_{D 3}+i_{D 5}+i_{D 7}$ and the load voltage at the full load condition. The current $i_{D 1}+i_{D 3}+i_{D 5}+i_{D 7}$ has less ripple current when compared to the ripple currents of $i_{D 1}+i_{D 3}$ and $i_{D 5}+i_{D 7}$. The measured switching frequencies with different input voltages and different load conditions are shown in Fig. 13. The high input voltage requires a low DC voltage gain. Therefore, the operating switching frequency increases if the input terminal voltage $V_{\text {in }}$ increases. A lower switching frequency is necessary if the load power is increased at the constant input terminal voltage. The measured efficiencies of the proposed converter under different load conditions are given in Fig. 14.

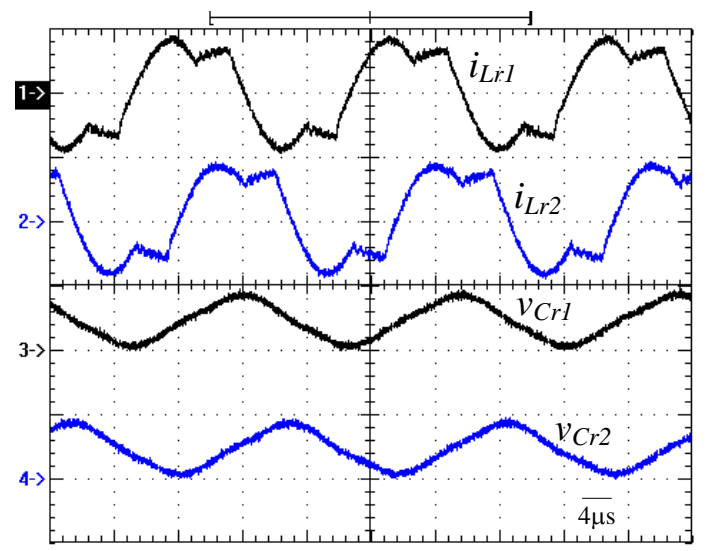

Fig. 9. Measured resonant inductor currents, $i_{L r 1}$ and $i_{L r 2}$, and resonant capacitor voltages, $v_{C r 1}$ and $v_{C r 2}$ at full load condition $\left[i_{L r 1}, i_{L r 2}: 50 \mathrm{~V} / \mathrm{div} ; i_{L r 1}, i_{L r 2}: 50 \mathrm{~A} / \mathrm{div}\right]$.

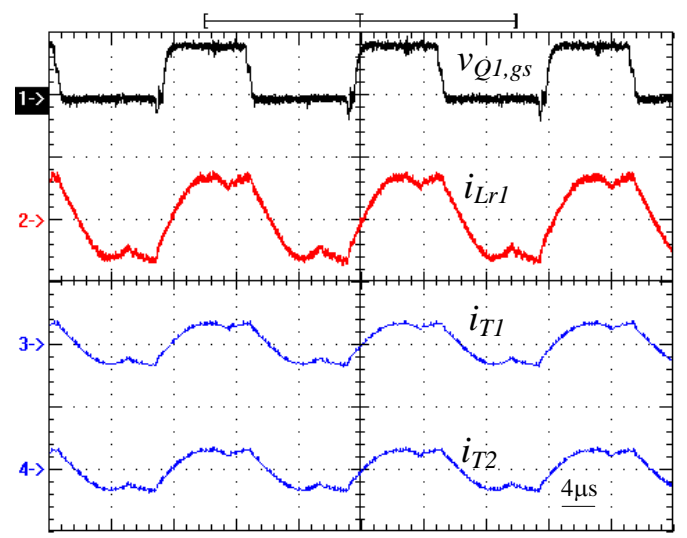

(a)

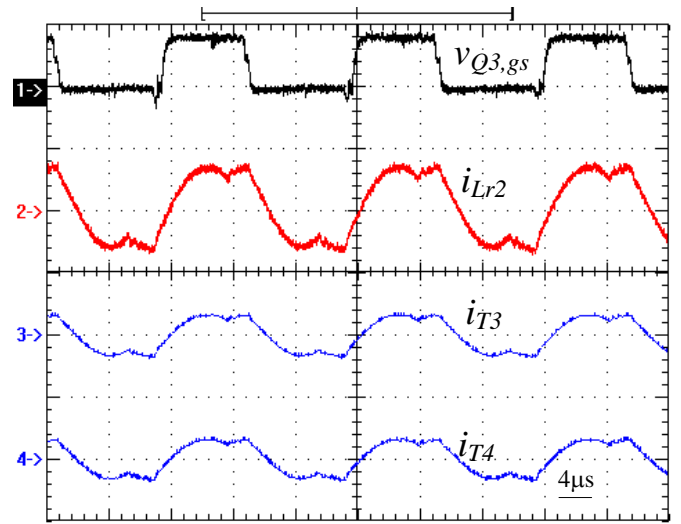

(b)

Fig. 10. Measured waveforms at nominal input voltage and full load (a) $v_{Q 1, g s}, i_{L r 1}, i_{T 1}$ and $i_{T 2}$ for converter module 1 (b) $v_{Q 3, g s}$, $i_{L r 2}, i_{T 3}$ and $i_{T 4}$ for converter module $2\left[v_{Q 1, g s}, v_{Q 3, g s}: 10 \mathrm{~V} / \mathrm{div} ; i_{L r 1}\right.$, $\left.i_{L r 2}, i_{T 1}, i_{T 2}, i_{T 3}, i_{T 4}: 50 \mathrm{~A} / \mathrm{div}\right]$.

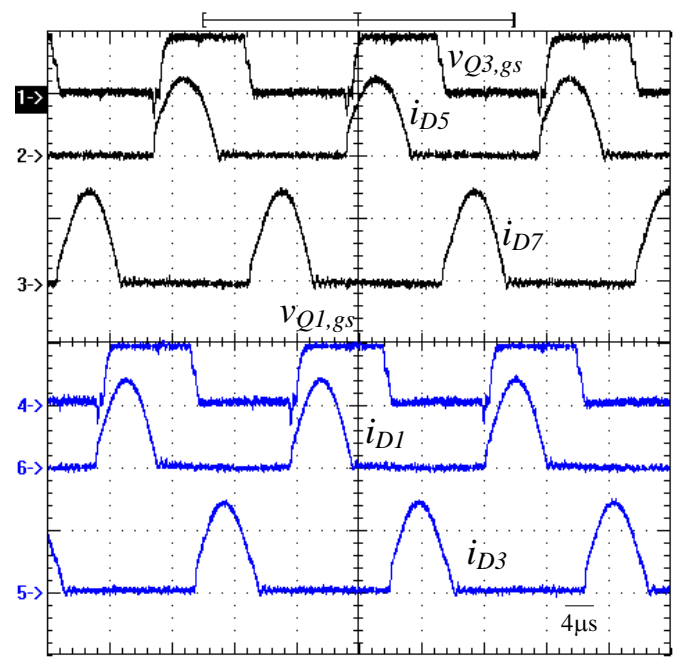

Fig. 11. Measured waveforms of the gate voltages, $v_{Q 1, g s}$ and $v_{Q 3, g s}$, and diode currents $i_{D 1}, i_{D 3}, i_{D 5}$ and $i_{D 7}$ at $V_{i n}=48 \mathrm{~V}$ and full load condition $\left[v_{Q 1, g s}, v_{Q 3, g s}: 10 \mathrm{~V} / \mathrm{div} ; i_{D 1}, i_{D 3}, i_{D 5}, i_{D 7}: 2 \mathrm{~A} / \mathrm{div}\right]$. 


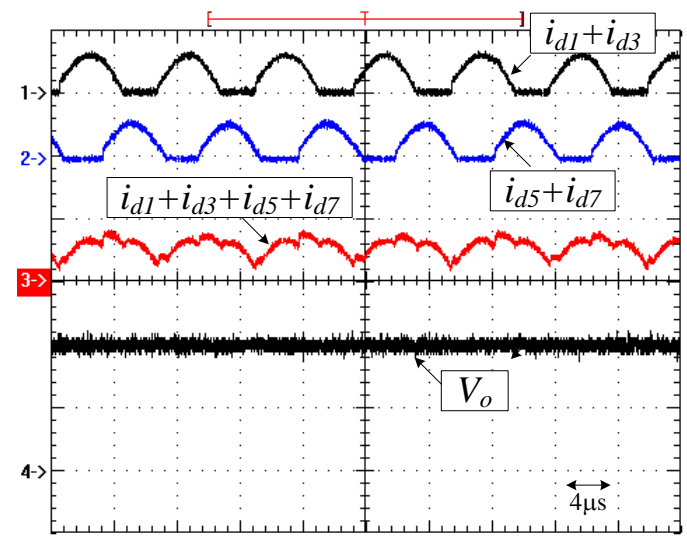

Fig. 12. Measured results of output currents $i_{D 1}+i_{D 3}$ and $i_{D 5}+i_{D 7}$ of two circuit modules, the resultant output current $i_{D 1}+i_{D 3}+i_{D 5}+i_{D 7}$ and output voltage $V_{o}$ at full load condition $\left[i_{D 1}+i_{D 3}, i_{D 5}+i_{D 7}, i_{D 1}+i_{D 3}+i_{D 5}+i_{D 7}: 5 \mathrm{~A} / \mathrm{div} ; V_{o}: 200 \mathrm{~V} / \mathrm{div}\right]$.

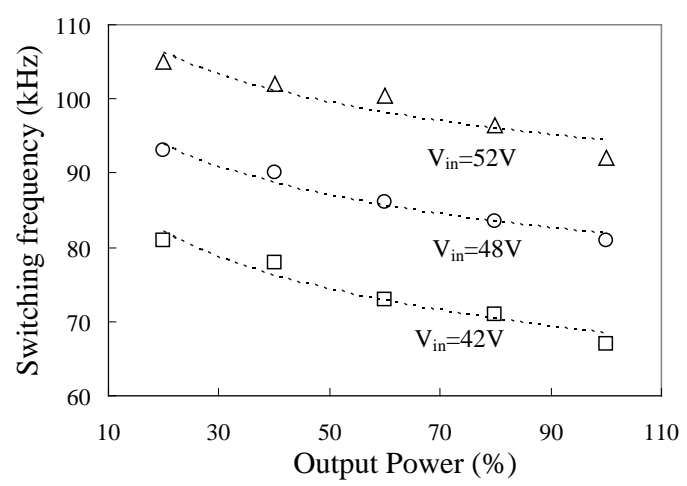

Fig. 13. Measured switching frequencies with different input voltages and load conditions.

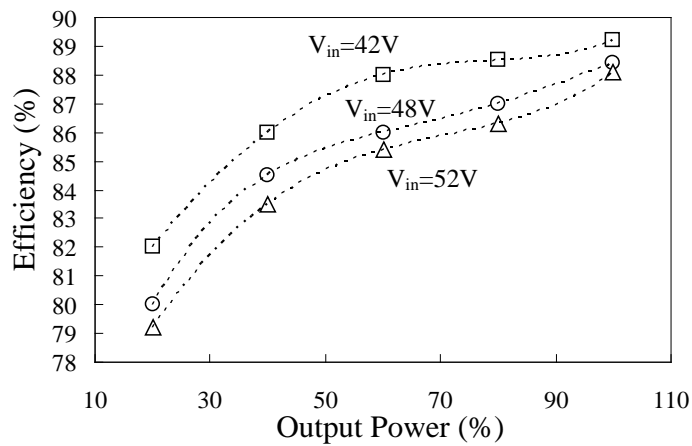

Fig. 14. Measured efficiencies of the proposed converter with different input voltages and load conditions.

\section{CONCLUSION}

This paper presents an interleaved series resonant converter to achieve ZVS turn-on for active switches and ZCS turn-off for rectifier diodes. Thus the switching losses and the reverse recovery losses of the power semiconductors are reduced and the ripple current at the output capacitor is also reduced. In each circuit module, two transformers are used to reduce the power rating of each magnetic core. The primary windings of the two transformers are connected in parallel and the secondary windings are connected in series in order to reduce and balance the current rating on each transformer. Thus the small size of the magnetic cores and reduced winding turns are adopted in each transformer. A full-wave diode rectifier is used in the high voltage side to clamp the voltage stress of the diodes at the output terminal voltage instead of at two times the output voltage as in a conventional center-tapped rectifier. The proposed converter can be used for low input voltage and high output voltage applications such as battery stack power conversion systems and renewable energy conversion systems. Finally experimental results are provided to verify the performance of the proposed converter.

\section{ACKNOWLEDGMENT}

The project is supported by the National Science Council of Taiwan, ROC, under Grant NSC101-2221-E-224-MY2.

\section{REFERENCES}

[1] P. Das, B. Laan, S. A. Mousavi, and G. Moschopoulos, "A Nonisolated Bidirectional ZVS-PWM active clamped DC-DC converter," IEEE Trans. Power Electron., Vol. 24, No. 2, pp. 553-558, 2009.

[2] I.-D. Kim, J.-Y. Kim, E.-C. Nho, and H.-G. Kim, “Analysis and design of a soft-switched PWM sepic DC-DC converter," Journal of Power Electron., Vol. 10, No. 5, pp. 461-467, Sep. 2010.

[3] B.-R. Lin, H.-K. Chiang, and C.-Y. Cheng, "Interleaved DC-DC converters with partial ripple current cancellation," Journal of Power Electronics, Vol. 12, No. 2, pp. 249-257, Mar. 2012.

[4] B. R. Lin and J. Y. Dong, “Analysis and implementation of an active clamping zero-voltage turn-on switching /zero-current turn-off switching converter," IET Proc. Power Electron., Vol. 3, No. 3, pp. 429-437, 2010.

[5] J.-H. Kim, Y.-C. Jung, S.-W. Lee, T.-W. Lee, and C.-Y. Won, "Power loss analysis of interleaved soft switching boost converter for single-phase PV-PCS," Journal of Power Electron., Vol. 10, No. 4, pp. 335-341, Jul. 2010.

[6] B.-R. Lin and C.-H. Tseng, "Analysis of parallel-connected asymmetrical soft-switching converter," IEEE Trans. Ind. Electron., Vol. 54, No. 3, pp. 1642-1653, Jun. 2008.

[7] J. C. P. Liu, N. K. Poon, B. M. H. Pong, and C. K. Tse, "Low output ripple dc-dc converter based on an overlapping dual asymmetric half-bridge topology," IEEE Trans. Power Electron., Vol. 22, No. 5, pp. 1956-1963, Sep. 2007.

[8] G. B. Koo, G. W. Moon, and M. J. Youn, "New zero-voltage-switching phase-shift full-bridge converter with low conduction losses,” IEEE Trans. Ind. Electron., Vol. 52, No. 1, pp. 228-235, Feb. 2005.

[9] H.-J. Chiu, Y.-K. Lo, T.-P. Lee, C.-C. Chuang, and S.-C. Mou, "A single-stage phase-shifted full-bridge AC/DC converter with variable frequency control," International Journal of Circuit Theory and Applications, Vol. 38, No. 8, pp. 867-879, 2010.

[10] S.-S. Hong, S.-K. Ji, Y.-J. Jung and C.-W. Roh, "analysis and design of a high voltage flyback converter with resonant 
elements,” Journal of Power Electron., Vol. 10, No. 2, pp. 107-114, Mar. 2010.

[11] A. K. S. Bhat, "Analysis and design of a modified series resonant converter," IEEE Trans. Power Electron., Vol. 8, No. 5, pp. 423-430, Oct. 1993.

[12] M. K. Kazimierczuk and N. Thirunarayan, "Class D voltage-switching inverter with tapped resonant inductor," IEE Proc. - Electric Power Applications, Vol. 140, No. 3, pp. 177-185, 1993.

[13] D. Czarkowski and M. K. Kazimierczuk, "Phase-controlled CLL resonant converter," in Proc. IEEE Applied Power Electronics Conference, pp. 432-438, 1993.

[14] M. K. Kazimierczuk, "Synthesis of phase-modulated dc/ac inverters and dc/dc converters," IEE Proc. - Electric Power Applications, Vol. 139, No. 4, pp. 387-394, 1992.

[15] M. K. Kazimierczuk and D. Czarkowski, Resonant Power Converters, Chap. 9 and 18, John Wiley \& Sons, 2011.

[16] Y. Gu, Z. Lu, L. Hang, Z. Qian, and G. Huang, “Three-level LLC series resonant DC/DC converter," IEEE Trans. Power Electron., Vol. 20, No. pp. 781-789,Jul. 2005;

[17] K. Jin, and X. Ruan, "Hybrid full-bridge three-level LLC resonant converter - A novel dc-dc converter suitable for fuel-cell power system,” IEEE Trans. Ind. Electron., Vol. 53, No. 5, pp. 1492-1503, Oct. 2006.

[18] D. Fu, Y. Liu, F. C. Lee, and M. Xu, “A novel driving scheme for synchronous rectifiers in LLC resonant converters," IEEE Trans. Power Electron., Vol. 24, No. 5, pp. 1321-1329, May 2009.

[19] K. H. Yi and G. W. Moon, "Novel two-phase interleaved LLC series-resonant converter using a phase of the resonant capacitor," IEEE Trans. Ind. Electron., Vol. 56, No. 5, pp. 1815-1819, May 2009.

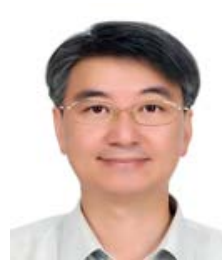

Bor-Ren Lin received his B.S. in Electronic Engineering from the National Taiwan University of Science and Technology, Taipei, Taiwan, in 1988, and his M.S. and Ph.D. in Electrical Engineering from the University of Missouri, USA, in 1990 and 1993, respectively. From 1991 to 1993, he was a Research Assistant with the Power Electronic Research Center, University of Missouri. Since 1993, he has been with the Department of Electrical Engineering, National Yunlin University of Science and Technology, Douliou, Taiwan, where he is currently a Professor. He has authored more than 200 published technical journal papers in the area of power electronics. His current research interests include power-factor correction, multilevel converters, active power filters, and soft-switching converters. Dr. Lin is an Associate Editor of the IEEE Transactions on Industrial Electronics, The Institution of Engineering and Technology Proceedings-Power Electronics and the Journal of Power Electronics. He was the recipient of Research Excellence Awards in 2004, 2005, 2007 and 2011 from the College of Engineering, National Yunlin University of Science and Technology. He received best paper awards from the IEEE Conference on Industrial Electronics and Applications 2007 and 2011, from the Taiwan Power Electronics 2007 Conference, and from the IEEE Power Electronics and Drive Systems 2009 Conference.

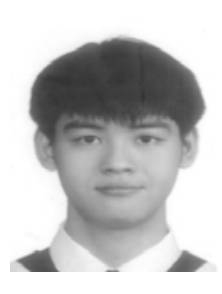

Sin-Jhih Shen received his M.S. in Electrical Engineering from the National Yunlin University of Science and Technology, Yunlin, Taiwan, in 2011. His current research interests include the design and analysis of power factor correction techniques, switching mode power supplies and soft switching converters. 